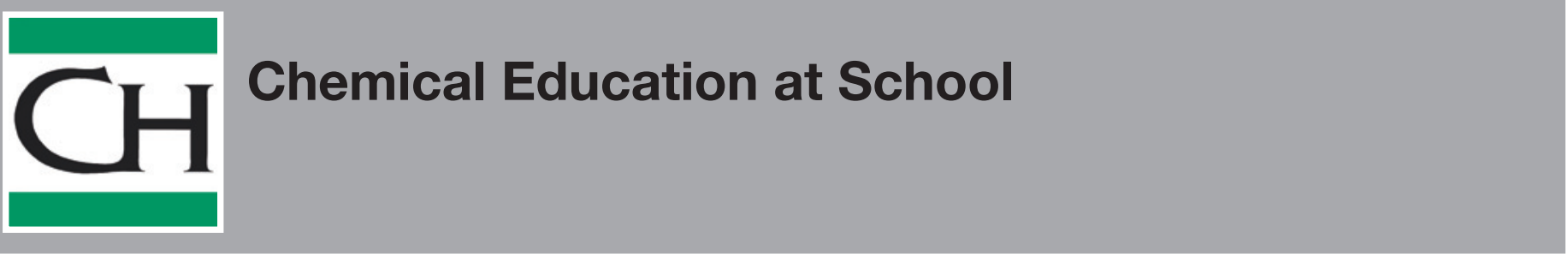

\section{BioChemie am Samstag 2010: Programm 2010}

Prof. Dr. Samuel Leutwyler (leutwyler@iac.unibe.ch) Direktor des Departements für Chemie und Biochemie

Universität Bern, Freiestrasse 3, 3012 Bern

La section de chimie/biochimie de l'université de Berne organise depuis plusieurs années une manifestation intitulée 'BioChemie am Samstag'. Il s'agit d'une série de conférences sur des thèmes variés liés à la chimie et la biochimie. Les conférences sont données en allemand. Bien que destinées primairement aux gymnasiens/lycéens, elles sont ouvertes à toutes personnes intéressées. Elles se déroulent le samedi matin de 10 h à 11 h30 dans l'aula principale (UG113) du département de chimie de l'Université de Berne (Freiestrasse 3, 3012 Berne) situé à 10 minutes à pied de la gare de Berne. Pour de plus amples d'informations, veuillez consulter le site web du département de chimie et biochimie de l'université de Berne (www.dcb.unibe.ch) ou contacter directement les conférenciers.

Der Fachbereich Chemie und Biochemie der Universität Bern organisiert seit mehreren Jahren eine Veranstaltungsreihe unter dem Titel 'BioChemie am Samstag'. Es handelt sich dabei um Vorträge zu verschiedenen Themen der Chemie und Biochemie. Die Vorträge finden in Deutsch statt. Obwohl diese vorwiegend für Gymnasiasten vorgesehen sind, können alle Interessierten daran teilnehmen. Die Vorträge finden jeweils am Samstagvormittag von 10 bis 11.30 Uhr im grossen Hörsaal (UG113) des Departements für Chemie und Biochemie, Freiestr. 3, 3012 Bern statt, welches zu Fuss in rund 10 Minuten vom Bahnhof Bern erreichbar ist. Weitere Informationen entnehmen Sie bitte der Homepage des Departements für Chemie und Biochemie der Universität Bern (www.dcb. unibe.ch) oder kontaktieren Sie direkt unsere Referenten.

\section{Samstag 4.9.2010}

\section{Brainstorm: Chemie im Gehirn}

Prof. Martin Lochner (martin.lochner@ dcb.unibe.ch)

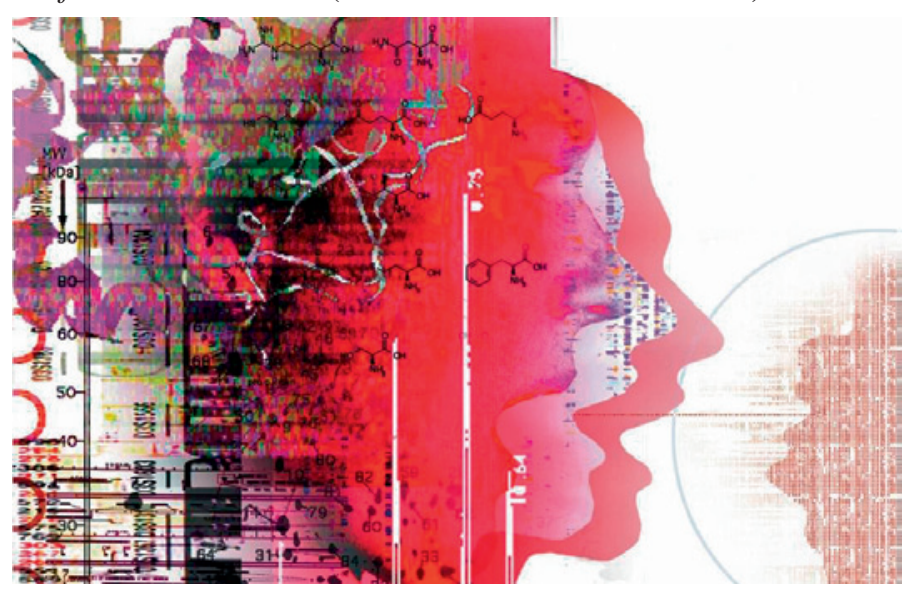

(C) 2010 M. Lochner
Millionen von chemischen Reaktionen laufen ständig in unserem Gehirn ab. Kleine Moleküle fungieren als chemische Botenstoffe und übertragen dadurch Nervensignale von einer Nervenzelle zur nächsten. Diese und andere chemische Aspekte des Gehirns werden im Vortrag diskutiert.

\section{Samstag 23.10.2010}

Chemische Experimente mit superschweren Atomen Prof. Andreas Türler (andreas.tuerler@psi.ch)

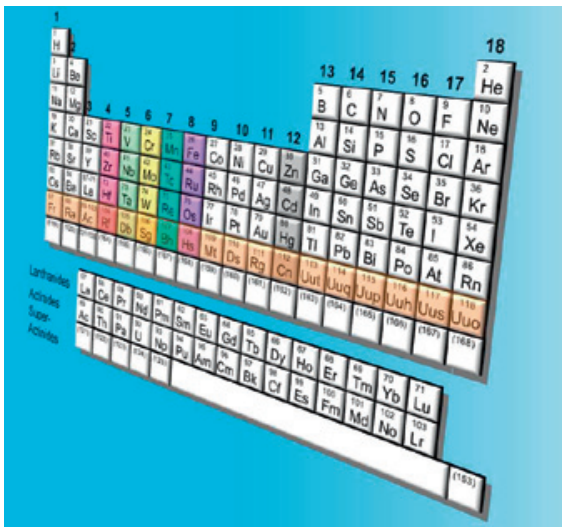

(c) 2010 A. Türler

Welches ist das schwerste Element im Periodensystem? Gibt es noch unentdeckte Element die womöglich sogar in der Natur vorkommen? Was sind die chemischen Eigenschaften der schwersten bekannten Elemente und kann man diese überhaupt untersuchen? Antworten auf diese Fragen gibt der Vortrag.

\section{Samstag 6.11.2010}

\section{Die Entstehung des Lebens}

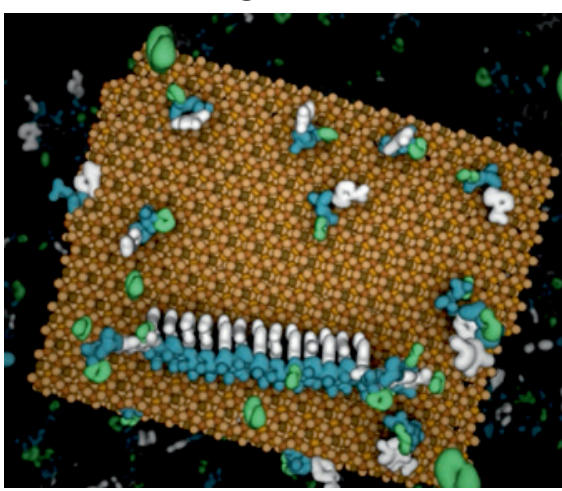

Prof. Oliver Mühlemann (oliver. muehlemann@dcb. unibe.ch)

Seit rund 200 Jahren wird die Frage nach der Entstehung des Lebens zunehmend auch mit naturwissenschaftlichen Methoden erforscht. Das aus heutiger wisCreative Commons License: Janet Iwasa senschaftlicher Sicht plausibelste Szenario für den graduellen

Übergang von unbelebter zu belebter Materie vor ca. 4 Milliarden Jahren wird in diesem Vortrag vorgestellt und diskutiert.

If you teach chemistry in a Swiss school or if you are involved with chemistry teaching at school level and would like to contribute to this column (a chemistry experiment, a project, etc.) please contact Dr. Roland Kunz: E-mail: kunz@oci.uzh.ch 


\section{Samstag 20.11.2010}

\section{Von 'A' wie Apfel bis ' $Z$ ' wie Zelle - unsere Lebensmittel im Visier}

Dr. Martina Vermathen (martina.vermathen@ioc.unibe.ch)

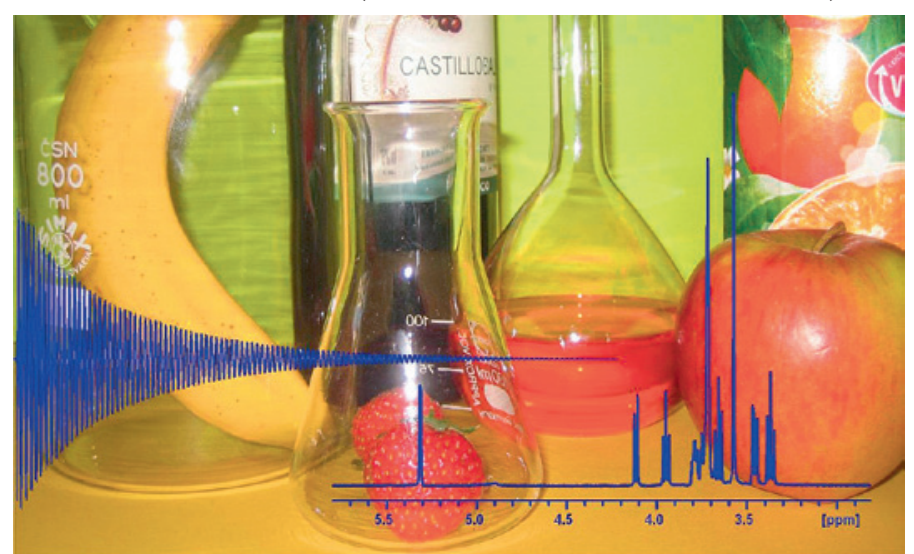

(C) 2010 M. Vermathen

Die Globalisierung der Wirtschaft hat sich besonders auch auf den Lebensmittelmarkt niedergeschlagen. Dieses stellt hohe Ansprüche an die chemischen Analysenverfahren, um die Qualität der Lebensmittel zu sichern und um zum Beispiel deren Herkunft nachzuweisen. In diesem Vortrag wird der Einsatz der Kernspinresonanz-Spektroskopie als eine der zukunftsweisenden Analysenmethoden für die Lebensmittelüberwachung vorgestellt.

\section{Samstag 4.12.2010}

\section{Chemische Spuren im ewigen Eis}

Prof. Margit Schwikowski (margrit schiwkowski@psi.ch)

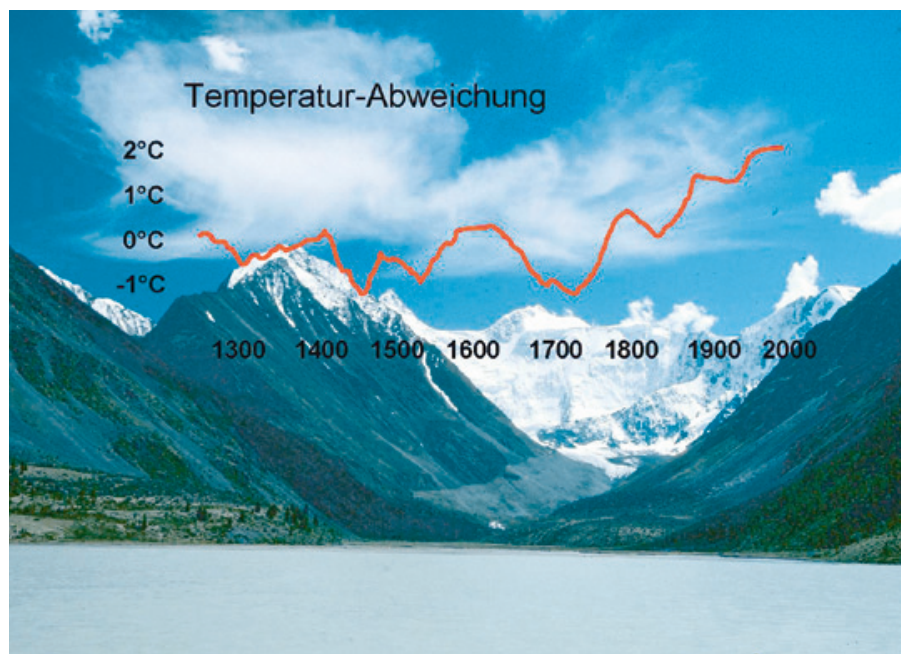

(C) 2010 M. Schwikowski

Im Eis der Hochgebirgsgletscher sind Informationen über Vulkanausbrüche, Saharastürme, Luftverschmutzung und sogar über die Temperaturen in den vergangenen Jahrhunderten gespeichert. Mit chemischen Analysen werden die Geheimnisse der eisigen Giganten zu Tage gefördert.

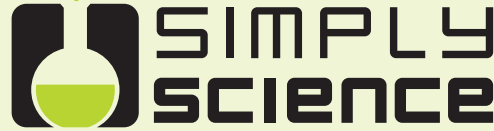

\section{AHA!-Frage: Was ist der Monsun?}

Antwort: Der Monsun ist eine grossräumige Luftbewegung, wobei die typischen Monsunwinde immer in eine Richtung blasen, die sie zweimal im Jahr ändern. Der Südwest-Monsun der Sommermonate bläst feuchte Meeresluft über Südostasien, die sich dann als sogenannter Monsunregen über das Festland entlädt. Auslöser für die Monsunströmung sind die grosse Landmasse Asiens und die Corioliskraft.

Sommer bedeutet in Europa Sonne und Wärme, unterbrochen von gelegentlichen Gewittern. Zumindest theoretisch - hier bei uns sind ja verregnete, kühle Sommer keine Seltenheit. Doch im Vergleich zu Ländern wie Indien ist die Schweiz im August 2010 knochentrocken. In Südostasien herrscht nämlich im Sommer Regenzeit. Der Monsunregen ist so stark, dass immer wieder Überschwemmungen wie zurzeit in Pakistan die Existenzgrundlage der Menschen wegschwemmen.

\section{Entstehung des Monsuns}

Ursache des Monsuns ist die riesige Landmasse Asiens. Im Sommer erwärmt sich das Festland sehr stark, und die warme Luft steigt besonders über dem tibetischen Hochland auf und bildet dort ein Tiefdruckgebiet. Dieses sommerliche Hitzetief ist Teil der sogenannten Innertropischen Konvergenzzone (ITC). Die ITC zieht um den ganzen Erdball, wie ein Band in der Nähe des Äquators. Sie ist die Zone der grössten Erwärmung. Durch die starke sommerliche Erwärmung des tibetischen Hochlands wird die ITC im Sommer über Südostasien jedoch weit nach Norden verschoben. Dadurch entsteht ein starkes Luftdruckgefälle zwischen dem subtropischen Hochdruckgebiet über dem Indischen Ozean (Südhalbkugel) und der ITC über dem tibetischen Hochland. Deshalb beginnt die
Diese Bild zeigt die Monsunwinde über Indien. Rot ist der Südwest-Monsun eingezeichnet, der über dem Äquator die Richtung ändert. Grün ist der Nordost-Monsun, und blau die ITC. (Bild: Wikimedia Commons).

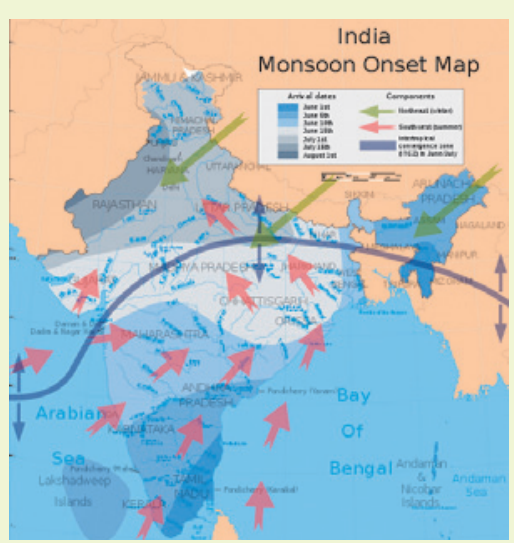

Luft von der Zone mit hohem Druck zur Zone mit niedrigem Druck zu fliessen. Der Südost-Monsunwind entsteht.

Sobald der Wind den Äquator überquert hat, wirkt die Corioliskraft auf ihn ein, und er ändert seine Richtung um $90 \mathrm{Grad}$, weht also nun gegen Westen, nach Indien und Pakistan bis nach China und Indonesien. Da der Monsunwind über dem Indischen Ozean feuchtwarme Luft aufgenommen hat, entlädt sich diese nun über dem Festland als Regen.

Im Winter ändern sich die Verhältnisse: Über dem innerasiatischen Festland steht kalte Luft, die ein Kaltlufthoch bildet. Der Luftdruck ist höher als im Süden Asiens, weshalb der Wind nun in die umgekehrte Richtung weht.

\section{Fluch und Segen}

Der Monsun ist eine Garantie für Regen, so dass die ganze Landwirtschaft in diesem Bereich nach dem Monsun ausgerichtet ist. Auch für die Trinkwasservorräte ist der Monsun von besonderer Bedeutung. Andererseits sind die Monsunwinde für immer wiederkehrende Naturkatastrophen verantwortlich. Im Winter bringen sie Dürre, im Sommer Überschwemmungen - so wie derzeit in Pakistan.

\section{Simplyscience.ch - die Website für Naturwissenschaften und Technik}

Geheimnisse des Alltags und wissenschaftliche Phänomene werden auf simplyscience.ch auf spannende Weise verständlich erklärt. Ob interessante Storys, faszinierende Kolumnen, coole Quiz mit tollen Preisen oder ausführliche Hintergrundinformationen in spannenden Dossiers, auf simplyscience.ch ist man immer auf dem Laufenden. Weitere Informationen: www.simplyscience.ch 